\title{
Comments on Bennett's model of somatic chromosome disposition
}

\author{
R. S. Callow
}

Department of Botany, The University, Manchester, M13 9PL.

\begin{abstract}
Bennett's model predicting specific spatial chromosomal arrangements of haploid genomes has been examined. The rationale is found to be obscure and the prima facie case unconvincing. A posteriori evidence does not withstand close scrutiny.
\end{abstract}

Cytologists have recently been invited to test a model predicting the mean spatial order of chromosomes in somatic nuclei (Heslop-Harrison and Bennett, 1983a). The rationale for such a prediction and the evidence so far presented in its favour are questioned in this paper. Examination is based on the following axioms: (i) the logical basis of a scientific hypothesis has to be open to scrutiny; (ii) tests must be directly related to predictions and (iii) any analysis of evidence should adhere to sound statistical procedures permitting objective interpretation.

Bennett's model assumes the separation of haploid complements within diploid nuclei and predicts a specific circular order of nonhomologous chromosomes, specified by the juxtaposition of most similar sized long or short arms and fixed about a single mismatch termed a discontinuity (Bennett, 1982). The prediction is a modification of an earlier model, according to which non-homologous chromosomes are attached telomerically in the arrangement giving the best approximation to a constant intercentromeric distance (Shchapova, 1971). Bennett's model gives the same centromeric order but operates by minimising the discrepancies in length between adjacent unlinked chromosome arms. Bennett considers his own model to be mechanically superior to Shchapova's but we are not told in what way. The specific mechanical and functional merits of the two hypotheses are not discussed.

What is Bennett's prima facie evidence? The assumption of genomic separation is based mainly on evidence from interspecific hybrids (Finch et al., 1981), supported by a small number of observations from established diploids (Bennett, 1983).
This may not be generally applicable to normal individuals and obviously cannot apply to the well known examples of somatic pairing, such as species of Drosophila and Aspergillus. Direct observations of somatic associations of nonhomologous chromosomes are rare (HeslopHarrison and Bennett, 1983a), perhaps the best being the study of specific telomeric attachments in Ornithogalum virens by Ashley (1979). Although this report is quoted by Bennett (1982) in support of his theory, it is again a special case; other species of Ornithogalum show somatic association of homologues and even secondary pairing (Therman, 1951). Where then does the prima facie evidence lead us? The proposition that somatic chromosome disposition is non-random (Avivi and Feldman, 1980; Maguire, 1984) is strongly contested (Darvey and Driscoll, 1972; John, 1976). Some observational inconsistencies may be resolved, as Bennett suggests, by referring to reconstructions of serial sections rather than squash preparations. The diversity already noted in Ornithogalum, however, could not be resolved in this way. The safest conclusion on the basis of background evidence appears to be that chromosome disposition is unlikely to conform to any single universal pattern.

$A$ priori we have been given no convincing basis, either logical or factual, for judging the plausibility of Bennett's hypothesis. We are left therefore with a posteriori observations. Let us examine those presented for Aegilops umbellulata $(2 n=14)$ which have been obtained from serial re-constructions of thin sections viewed by electron microscopy. Information on ten root-tip cells has been presented: five fixed directly and five 
pretreated with iced water. Individual metaphase chromosomes were identified on the basis of arm volume ratio and percentage of total mitotic volume. Evidently every effort has been made to preserve the three-dimensional structure of each cell and maintain accuracy, although the possibility of mis-identification remains a problem for certain chromosomes. Three-dimensional coordinates were obtained for each centromere and subjected to two distinct analytical procedures: generalised Procrustes (Heslop-Harrison, 1983) and ordination of summed intercentromeric distances (Heslop-Harrison and Bennett, 1983a,b). The latter approach has also been applied to centromeric data from Hordeum vulgare var. Tuleen 346 and Secale africanum (Bennett, 1982). Before considering these approaches, we are aware that whatever their outcome they refer to the disposition of centromeres rather than the relationship between arms predicted in Bennet's model. At best they cannot distinguish between the schemes proposed by Bennett and Shchapova.

Procrustes analysis is so named (after the tyrant defeated by Theseus; c.f. Oldfather, 1939, p. 5) because it "lends itself to the brutal feat of making almost any data fit almost any hypothesis" (Hurley and Cattell, 1962). It is concerned with the manipulation of multidimensional co-ordinates so as to achieve consensus positions for sets of configurations. Gower (1975) has devised a method for partitioning the variation between and within such sets but his analysis of variance has no degrees of freedom and so cannot as yet be used as a test of significance.

Before being subjected to Procrustes, the three dimensional co-ordinates of centromeres of Aegilops umbellulata were rotated so as to achieve minimal variation in the third axis, thereby obtaining a metaphase plate in polar view (HeslopHarrison, 1983). Two haploid sets were then identified from each plate, presumably following the assumption of genomic separation, although we are not informed of the criteria employed. Threedimensional co-ordinates of the centromeres in each of twenty haploid sets obtained in this way were subjected to Gower's Procrustes analysis and separate consensus positions derived for the centromeres of each chromosome type. The resulting geometrical pattern has not been tested statistically nor indeed is it obviously in accordance with the predicted arrangement (Heslop-Harrison, 1983, fig. 3).

Let us now examine the ordination technique which has been applied to centromeric co-ordinates and which is advocated as a general cytologi- cal method (Heslop-Harrison and Bennett, 1983a). The procedures may be summarised in four stages as follows:

(1) With two homologues as fixed reference points, each of the $(x-1) ! / 2$ unidirectional circular permutations of the remaining $x-1$ chromosomes in each basic haploid set is ranked separately for each diploid cell, according to the sum of the sides of the polygon whose corners are marked by the centromeres in the prescribed order. For this purpose, the $2^{x-1}$ pairwise orientations of homologous chromosomes between the 2 haploid sets of a diploid are so adjusted that the accumulated intercentromeric distance is minimised.

(2) The unidirectional circular permutations, termed orders, are listed in order of ranks summed over all the cells. Lowest summed ranks have the smallest average accumulated intercentromeric distance and are termed "best". The implied definition of best appears to be entirely teleological.

(3) If the permutation predicted a priori on the Bennett model occurs in the top 5 per cent of orders it is said to be "significantly better" than "the others". If in the top 1 per cent, then it is highly significantly better.

(4) Cells which show great departure from the predicted pattern can, apparently, be arbitrarily removed from the analysis.

Stages I and 2 attempt to constrain the observations, as far as possible, to comply with Bennett's hypothesis. In the process, measurements have been down-graded from a ratio to an ordinal scale, with concomitant loss of information ( $\mathrm{cf}$. Conover, 1971, pp. 66-67). Another disturbing property of this approach, from a statistical viewpoint, is that each cell must have the same average rank, equal to $(k+1) / 2$, over all $k$ orders so that any overall differences between cells are inevitably completely suppressed. Moreover, if a particular spatial order does not have exactly the same rank in every cell, it will decrease the likelihood of uniform cellular ranking for the remaining spatial orders, since where it occupies high ranks other orders are more likely to be low and vice versa. A fixed number of rankings within cells, therefore, is bound to generate spurious negative correlations between cells. It is for this reason that ranking statistics are usually based on collective rather than separate ordination of samples (Conover, 1971, p. 223). Thus in stages 1 and 2 cellular variation is not only ignored, it is distorted beyond analytical recognition. 
Stage 3 indicates that Bennett and HeslopHarrison have a rather unusual approach to statistical inference, an approach of which we should be extremely wary. Instead of computing the probability with which an observed deviation from expectation is likely to occur on the basis of chance alone, we are urged to decipher which prescribed arrangement of chromosomes is significantly "better" than all the others and to do this when we have no direct evidence, on the authors' own admission, that such arrangements even exist. Furthermore, we are given no biological explanation of the criteria by which one arrangement is to be judged better than another.

The philosophy behind this novel significance test is certainly difficult to follow. The method is supposed to constitute an independent test, because the best order is predicted a priori and then tested against, what are termed, actual orders. Such a supposition is surely unmerited, since all the possible orders are at present hypothetical and not only classified but also partly derived according to the same a priori specifications as the prediction. Bennett's predicted order may well be best according to his criteria but this would be a geometrical property not a statistical one. The predicted order has yet to be actually revealed in mitotic cells by comparison of three-dimensional co-ordinates of the chromosomes in a large number of nuclei.

Another disturbing aspect of Heslop-Harrison and Bennett's approach is their comment (HeslopHarrison and Bennett, $1983 a$, p. 221 ) on the relative importance of low cell number and low chromosome number. The former is not considered a serious drawback while the latter is thought to prevent a satisfactory statistical analysis from being conducted, because of the low number of possible orders if a haploid set has fewer than six chromosomes. Surely the reverse is true in each case. Adequate sample sizes are obviously indispensable for statistical analysis and cells with low chromosome numbers must generally be more amenable to cytological study than those with high numbers: a conclusion reached by earlier students of somatic chromosome disposition (Ashley, 1979). Taking the advice of Bennett and HeslopHarrison at face value, it should be possible to carry out a significance test on chromosome disposition in a single nucleus, provided it has a high chromosome number. Indeed, cellular variation is not only unmeasured by these authors but is considered of such little consequence that unwelcome cells seem to be removable at will. Looking at some of the evidence as presented for Aegilops umbellu- lata (Heslop-Harrison and Bennett, 1983b, table 4 ), we see that the orders which minimise cellular variation in rank and hence appear to be most representative of the biological material are those which have the greatest accumulated intercentromeric distances, despite the best endeavours of the ordination procedure to reduce such distances.

A knowledge of somatic chromosome disposition would obviously have important cytogenetical implications, for example in predicting categories of spontaneous structural change. Bennett (1983) has even suggested that both C-bands and sets of non-allelic genes of similar phenotype, termed paralogous, may tend to be clustered on adjacent but unlinked heterologous arms. We are left to infer that spatial proximity brings its own advantage but, here again, the line of reasoning is not made obvious. Evidence is presented in the form of an analysis of the chromosomal locations of 24 pairs and 9 triplets of paralogous genes of $\mathrm{Zea}$ mays. Maize is assumed to be an allotetraploid $(2 n=4 x=20)$ with chromosomes arranged in four subsets of 5 heterologues. The frequencies, with which sets of paralogous genes occur on putative homoeologous chromosomes or on heterologues adjacent in the Bennett model, are compared with their "random" expectations. These frequencies are greater than expected and we are told that the differences are significant, although the statistical procedure is not identified (Bennett, 1983, table 3 ).

In Bennett's own words (1983, p. 78), this result "illustrates the power of the model in revealing hitherto unknown details of karyotype architecture, in this case suggesting that Zea mays is a tetraploid and indicating which chromosomes are homoeologous". Even if the distribution of paralogous genes were proven to be non-random, a matter to which we will return, such circular reasoning could not be justified. Our knowledge of so called paralogous genes does not, at present, allow us to infer homoeologous relationships or even tetraploidy let alone patterns of spatial disposition. On the contrary, such evidence as is available indicates that maize is a true diploid (Mangelsdorf, 1974, p. 72).

The postulate that paralogous genes cluster on adjacent but unlinked chromosome arms is not tested in the analysis, since loci are only resolved to whole chromosomes. At the same time, instances of two linked paralogous genes are excluded unless a third unlinked paralogue is present (e.g., Liguleless). Moreover linkage does not occupy a separate category in Bennett's analysis yet it is obviously a more reliable indicator of proximity than any notional homoeology or heterologous adjacency. 
Of course proximity per se may not be important but, in the absence of any clear rationale, it is perhaps the best inference we can make.

Two particular features of Bennett's table 3 give cause for concern. Firstly the expected frequency of homoeology amongst three paralogous genes $(1 / 8)$ is only marginally greater than that amongst just two paralogues (1/9) yet 3 pairwise combinations are possible in the former case but only one in the latter. Secondly, while the three categories of homoeology, adjacency and nonadjacency are distinct in the case of two paralogous genes, when three paralogues are considered they overlap. For example, two genes may be on homoeologous chromosomes and a third on an adjacent heterologue. In the case of three paralogous genes, therefore, the three categories should not have proportions which sum to one, as is the case in Bennett's table. In view of these reservations is the distribution of paralogous genes in maize non-random as suggested?

I have turned to Bennett's sources (Neuffer and Coe, 1974; Goodman et al., 1980) and made my own analysis whilst adhering to the Bennett chromosome model. I selected 29 pairs and 10 triplets of paralogous genes, including examples of linkage and only omitting unmapped genes and those of deviant phenotype (Appendix A). Expected values were derived to include linkage (Appen$\operatorname{dix} B$ ) and the data for sets of two and three paralogous genes were combined, in goodness-offit tests, because of the small sample sizes. To avoid the problem of overlapping categories, separate tests have been employed for linkage, "homoeology" and "adjacency". Each analysis shows no obvious heterogeneity between sets of two and three paralogous genes and in each any deviation from randomness is non-significant (table 1).

Returning to the axioms listed at the beginning of this paper, I can find no information on the causal basis of Bennett's hypothesis, no persuasive arguments in its favour and no evidence which bears close scrutiny. This is unfortunate because the idea has interesting genetical implications (Bennett, 1981) and has already provided a stimulus to studies of chromosome disposition. Those cytologists who have been encouraged in this respect should, however, realise that they are working in a minefield of scientific inference and that statistical validation of their observations may require enormous sample sizes, especially if their specimens have high chromosome numbers.

Acknowledgements I am grateful for the valuable advice of Professors J. L. Jinks and Sir Kenneth Mather and Drs J. S. Gale and G. H. Jones in the Genetics Department at Birmingham University and of my colleague Dr I. Gladwell in the Mathematics Department at Manchester.

\section{REFERENCES}

ASHLEY, T. 1979. Specific end to end attachment of chromosomes in Ornithogalum virens. J. Cell Sci., 38, 357-367.

AVIVI, l. ANI) FELIDMAN. M. 1980. Arrangement of chromosomes in the interphase nucleus of plants. Hum. Genet. $55,281-295$.

BENNETT, M. D. 1981. The spatial order of barley chromsomes. Barley genetics IV. Proc. 4th int. Barley Genet. Symp. 1981. pp. 75I-757. International Barley Genetics Symposium, Edinhurgh.

BENNETT, M. D. 1982. Nucleotypic basis of the spatial ordering of chromosomes in eukaryotes and the implications of the order for genome evolution and phenotypic variation. In Genome evolution, pp. 239-261. G. A. Dover and R. B. Flavell, (eds.) Academic Press, London and New York.

Table 1 Distribution of sets of paralogous genes amongst the chromosome complement of Zea mays arranged according to Bennett (1983) (see Appendix A), compared with predictions (in parentheses) of randomness (Appendix B)

\begin{tabular}{|c|c|c|c|c|c|c|c|}
\hline \multirow[b]{2}{*}{ Test of: } & \multicolumn{2}{|c|}{ Linkage } & \multicolumn{2}{|c|}{ "Homoeology" } & \multicolumn{2}{|c|}{ "Adjacency" } & \multirow{2}{*}{$\begin{array}{l}\text { Total } \\
\text { sets }\end{array}$} \\
\hline & $2 \mathrm{~L}$ & remainder & $2 \mathrm{H}$ & remainder & $2 \mathrm{~A}$ & remainder & \\
\hline \multicolumn{8}{|c|}{ paralogues/set } \\
\hline 2 & $\begin{array}{c}4 \\
(2 \cdot 9)\end{array}$ & $\begin{array}{l}25 \\
(26 \cdot 1)\end{array}$ & $\begin{array}{c}4 \\
(2 \cdot 9)\end{array}$ & $\begin{array}{l}25 \\
(26 \cdot 1)\end{array}$ & $\begin{array}{c}6 \\
(4 \cdot 6)\end{array}$ & $\begin{array}{l}23 \\
(24 \cdot 4)\end{array}$ & 29 \\
\hline 3 & $\begin{array}{l}4 \\
(2 \cdot 7)\end{array}$ & $\begin{array}{c}6 \\
(7 \cdot 3)\end{array}$ & $\begin{array}{l}5 \\
(2 \cdot 7)\end{array}$ & $\begin{array}{c}5 \\
(7 \cdot 3)\end{array}$ & $\begin{array}{l}5 \\
(3 \cdot 6)\end{array}$ & $\begin{array}{c}5 \\
(6 \cdot 4)\end{array}$ & 10 \\
\hline combined & $\begin{array}{l}8 \\
(5 \cdot 6)\end{array}$ & $\begin{array}{l}31 \\
(33 \cdot 4)\end{array}$ & $\begin{array}{l}9 \\
(5 \cdot 6)\end{array}$ & $\begin{array}{l}30 \\
(33 \cdot 4)\end{array}$ & $\begin{array}{l}11 \\
(8 \cdot 2)\end{array}$ & $\begin{array}{l}28 \\
(30 \cdot 8)\end{array}$ & \multirow[t]{2}{*}{39} \\
\hline $\begin{array}{l}\chi_{11]}^{2} \\
\text { probability }\end{array}$ & \multicolumn{2}{|c|}{$\begin{aligned} & 1 \cdot 201 \\
> & 0.2\end{aligned}$} & \multicolumn{2}{|c|}{$\begin{aligned} & 2 \cdot 410 \\
> & 0.1\end{aligned}$} & \multicolumn{2}{|c|}{$\begin{aligned} & 1 \cdot 211 \\
> & 0.2\end{aligned}$} & \\
\hline
\end{tabular}


BENNETT, M. D. 1983. The spatial distribution of chromosomes. Kew Chromosome Conference II (ed.) P. E. Brandham \& M. D. Bennett, pp. 71-79. London, George Allen \& Unwin CONOVER, W. J. 1971. Practical nonparametric statistics. Wiley, New York.

DARVEY, N. L. ANI) DRISCOLL, C. J. 1972. Evidence against somatic association in hexaploid wheat. Chromosoma, 36, 140-149.

FINCH, R. A., SMITH, J. B. AND BENNETT, M. D. 1981. Hordeum and Secale mitotic genomes lie apart in a hybrid. J. Cell Sci., 52, 391-403.

GOODMAN, M. M., STUBER, C. W., NEWTON, K., AND WEISSINGER, H. H. 1980. Linkage relationships of 19 enzyme loci in maize. Genetics, 96, 697-710.

Gower, J. C. 1975. Generalized Procrustes analysis. Psychometrika, 40, 33-51.

HESLOP-HARRISON, J. S. 1983. Chromosome disposition in Aegilops umbellulata. Kew Chromosome Conference II (ed.) P. E. Brandham \& M. D. Bennett, pp. 63-70. London, George Allen \& Unwin.

HESLOP-HARRISON, J. S. AND BENNETT, M. D. $1983 a$. Prediction and analysis of spatial order in haploid chromosome complements. Proc. R. Soc. Lond. B, 218, 211-223.
HESLOP-HARRISON, J. S. \& BENNETT, M. D. $1983 b$. The spatial order of chromosomes in root-tip metaphases of Aegilops umbellulata. Proc. R. Soc. Lond. B, 218, 225-239.

HuRley, J. R. AND CATTEL, R. B. 1962. The Procrustes program: producing direct rotation to test a hypothesized factor structure. Behavioral Science, 7, 258-262.

JOHN, B. 1976. Myths and mechanisms of meiosis. Chromosoma, $54,295-325$.

MAGUIRE, M. P. 1984. The mechanism of meiotic homologue pairing. J. theor. Biol., 106, 605-615.

MANGELSDORF, P. C. 1974. Corn: it's origin, evolution and improvement. Harvard, Cambridge, Massachusetts.

NEUFFER, M. G. AND COE, E. H. 1974. Corn (Maize) in Handbook of Genetics 2, pp. 3-30. ed. R. C. King. Plenum, New York and London.

OLDFATHER, C. H. 1939. The library of history of Diodorus of Sicily III. The Loeb Classical Library. Heinemann, London.

SHCHAPOVA, A. I. 1971. On the karyotype pattern and the chromosome arrangement in the interphase nucleus. (Russian with English summary) Tsitologia, 13, 1157-1164.

THERMAN, E. 1951. Somatic and secondary pairing in Ornithogalum. Heredity, 5, 253-269. 
APPENDIX A

Table IA Chromosomal locations of 29 pairs and 10 triplets of paralogous genes in the gametic complement of Zea mavs $(n=[2 \times ?]=10)$, displayed according to Bennett's (1983) theory of somatic disposition and homoeology (1 and 5, 4 and 2 etc.). Numbers in parentheses are abbreviations for non-allelic genes of similar phenotype: thus ba and $b a 2$ become $b a(2)$ while $A d h 1$ and $A d h 2$ are shortened to $A d h$ 1(2). Data for genes symbolized in Roman type are from Neuffer and Coe (1974), those in italics being due to Goodman et al. (1980)

\begin{tabular}{|c|c|c|c|c|c|c|c|c|c|c|c|c|c|c|c|c|c|c|c|c|c|c|}
\hline \multirow[b]{3}{*}{ Genes } & \multicolumn{18}{|c|}{ Chromosome } & & \multicolumn{3}{|c|}{ Grouping } \\
\hline & \multicolumn{2}{|c|}{1} & \multicolumn{2}{|c|}{4} & \multicolumn{2}{|c|}{9} & \multicolumn{2}{|c|}{6} & \multicolumn{2}{|c|}{8} & \multicolumn{2}{|c|}{5} & \multicolumn{2}{|c|}{2} & \multicolumn{2}{|c|}{3} & \multicolumn{2}{|c|}{7} & 10 & \multirow[b]{2}{*}{$2 \mathrm{~L}$} & \multirow[b]{2}{*}{$2 \mathrm{H}$} & \multirow[b]{2}{*}{$2 \mathrm{~A}$} \\
\hline & $\mathrm{s}$ & 1 & 1 & $\mathrm{~s}$ & s & 1 & 1 & $s$ & $s$ & 1 & $s$ & 1 & 1 & s & $s$ & 1 & 1 & s & s 1 & & & \\
\hline$a(2)$ & $\cdot$ & . & . & . & . & . & . & . & . & . & $*$ & . & . & . & . & $*$ & . & . & . & - & - & 一 \\
\hline $\mathrm{ba}(2)$ & . & . & . & . & . & . & . & . & . & . & . & . & $x$ & $x$ & . & $*$ & . & . & . & - & - & 1 \\
\hline $\mathrm{Bf}, \mathrm{bf} 2$ & . & . & . & . & . & $*$ & . & . & . & . & . & . & . & . & . & . & . & . & $*$ & - & - & - \\
\hline $\mathrm{bt}(2)$ & . & . & . & $*$ & . & . & . & . & . & . & . & $*$ & . & . & . & . & . & . & . & - & - & - \\
\hline bz.(2) & . & $*$ & . & . & $*$ & . & . & . & $\cdot$ & . & & $\cdot$ & . & . & . & . & . & $\cdot$ & . & - & - & - \\
\hline $\operatorname{cp}(2)$ & . & $\cdot$ & . & $\cdot$ & $\cdot$ & . & . & . & . & . & . & $\cdot$ & . & . & . & . & . & \# & . & 1 & - & - \\
\hline $\operatorname{de}(16)$ & . & . & . & \# & . & . & . & . & . & . & . & . & . & . & . & . & . & . & . & 1 & - & - \\
\hline $\mathrm{fl}(2)$ & . & . & . & $*$ & . & . & . & . & . & . & . & $\cdot$ & . & $*$ & . & $\cdot$ & $\cdot$ & $\cdot$ & . & - & 1 & - \\
\hline$g(2)$ & . & . & . & . & . & . & . & . & . & . & . & . & . & . & . & . & $x$ & $\times$ & * & - & - & 1 \\
\hline $\operatorname{gs}(2)$ & . & $*$ & . & . & . & . & . & . & . & . & . & . & . & $*$ & . & . & $\cdot$ & $\cdot$ & . & - & - & - \\
\hline $\mathrm{hm}(2)$ & . & $*$ & . & . & $x$ & $x$ & . & . & . & . & . & . & . & . & . & . & . & . & . & - & - & - \\
\hline $\mathrm{j}(2)$ & . & . & * & . & . & . & . & . & . & $*$ & . & . & . & . & . & . & . & . & . & - & - & - \\
\hline $\operatorname{lo}(2)$ & . & . & . & $*$ & $*$ & . & . & . & . & . & . & . & . & . & . & . & . & . & . & - & - & 1 \\
\hline na(2) & $\cdot$ & $\cdot$ & . & . & . & . & . & . & . & . & . & $*$ & . & . & . & $*$ & . & . & . & - & - & - \\
\hline $\mathrm{pb}(4)$ & . & . & . & . & . & . & $*$ & $x$ & $\cdot$ & . & . & $\cdot$ & . & $\cdot$ & . & $\cdot$ & . & $\cdot$ & . & 1 & - & - \\
\hline $\mathrm{R}(2)$ & . & . & . & . & . & . & . & . & . & . & . & . & . & $*$ & . & . & . & . & $*$ & - & - & - \\
\hline $\operatorname{rd}(2)$ & . & $*$ & . & . & . & . & $*$ & . & . & . & . & . & . & . & . & . & . & . & . & - & - & - \\
\hline $\mathrm{Rf}(2)$ & . & . & . & . & $x$ & $\times$ & . & . & . & . & . & . & . & . & $*$ & . & . & . & . & - & 1 & - \\
\hline $\mathrm{sp}(2)$ & . & . & . & $\ddot{*}$ & . & & . & . & . & . & . & . & . & . & . & . & . & . & $*$ & - & - & - \\
\hline $\operatorname{su}(2)$ & . & . & . & $*$ & . & . & * & . & . & . & . & $\cdot$ & . & . & . & . & . & $\cdot$ & . & - & - & - \\
\hline $\operatorname{tn}(2)$ & . & . & . & $\cdot$ & . & . & . & . & $\cdot$ & . & $\times$ & $\times$ & $\cdot$ & . & $\cdot$ & $\cdot$ & . & . & $x$ & - & - & - \\
\hline $\mathrm{Tp}(2)$ & . & . & . & . & . & . & . & . & $\cdot$ & . & $\cdot$ & $\cdot$ & . & . & . & . & $*$ & . & $*$ & - & - & 1 \\
\hline $\operatorname{yg}(2)$ & . & . & . & . & $*$ & . & . & . & . & . & $x$ & $x$ & . & . & . & . & . & . & . & - & - & - \\
\hline ys (3) & . & . & . & . & . & . & . & . & . & . & $\cdot$ & $*$ & . & . & * & . & . & . & . & - & - & - \\
\hline$A d h 1(2)$ & . & $*$ & . & * & . & . & . & . & . & . & . & . & . & . & . & . & . & . & . & - & - & 1 \\
\hline Cat1(2) & $*$ & . & & . & . & . & . & . & . & . & $*$ & . & . & . & . & . & . & . & . & - & 1 & - \\
\hline $\operatorname{Got} 2(3)$ & . & . & . & . & . & . & . & . & . & . & $*$ & * & . & . & . & . & . & . & . & 1 & - & - \\
\hline $\operatorname{Idh} \mathbf{1}(2)$ & . & $\cdot$ & . & . & . & . & $*$ & . & $x$ & $x$ & . & & . & . & . & $\cdot$ & . & . & . & - & - & 1 \\
\hline$P g m 1(2)$ & . & $*$ & . & . & $\cdot$ & . & $\cdot$ & . & $\cdot$ & $\cdot$ & $*$ & . & $\cdot$ & . & . & $\cdot$ & . & . & . & - & 1 & - \\
\hline $\operatorname{Dt}(2 \mathrm{~m} 3)$ & . & . & . & . & * & . & * & . & . & . & . & . & . & . & $\cdot$ & . & $*$ & . & . & - & 1 & 1 \\
\hline $\lg (2,3)$ & . & . & . & . & . & . & . & . & . & . & . & . & . & $*$ & $*$ & $*$ & . & . & . & 1 & - & 1 \\
\hline$o(2,5)$ & . & . & $*$ & . & . & . & . & . & . & . & . & . & . & . & $\cdot$ & . & $*$ & $*$ & . & 1 & - & - \\
\hline $\mathrm{ra}(2,3)$ & . & . & $x$ & $x$ & . & . & . & . & . & . & . & . & . & . & $*$ & · & $*$ & . & . & - & - & 1 \\
\hline $\mathrm{Rp}(3,4)$ & . & . & . & $*$ & . & . & . & . & . & . & $\cdot$ & $\cdot$ & . & . & . & $*$ & . & . & $*$ & - & - & - \\
\hline $\operatorname{sh}(2,4)$ & . & . & . & . & $*$ & . & . & . & . & . & $\times$ & $x$ & . & . & . & $*$ & . & . & . & - & 1 & - \\
\hline $\operatorname{sr}(2,3)$ & $*$ & . & . & . & . & . & . & . & . & . & . & . & . & . & . & . & . & . & $*$ & 1 & - & - \\
\hline$Y(y 8,9)$ & . & . & . & . & . & . & * & . & . & . & . & . & . & . & . & . & . & $*$ & $*$ & - & 1 & 1 \\
\hline zb $3(4,6)$ & * & . & $*$ & . & . & . & . & . & . & . & . & $*$ & . & . & . & . & . & . & . & - & 1 & 1 \\
\hline$A m p l(2,3)$ & $\cdot$ & \# & $\cdot$ & . & . & . & . & . & . & . & $*$ & . & . & . & . & . & . & . & . & 1 & 1 & - \\
\hline
\end{tabular}

s: short arm, l: long arm, *: gene locus, \# : two gene loci, $x$ : locus not mapped to individual arm, $2 \mathrm{~L}$ : two linked loci, $2 \mathrm{H}$ : two loci on putative homoeologues, $2 \mathrm{~A}$ : two loci on putatively adjacent heterologues.

\section{APPENDIX B}

Here we consider probabilities governing the random distribution of paralogous genes to linked (L), putatively homoeologous $(\mathrm{H})$ or heterologous adjacent (A) chromosomal locations in the gametic complement of Zea mays, arranged in two subsets of 5 heterologues according to Bennett (1983) (c.f. table 1A). These expressions have been checked against the complete enumeration of all 100 possible distributions for two paralogous genes amongst ten chromosomes and all 1000 possibilities for three paralogous genes.

1. Distribution of 2 paralogous genes

\subsection{Linkage}

$$
P[2 \mathrm{~L}]=\frac{1}{10}
$$




\section{2. "Homoeology"}

$$
P[2 \mathrm{H}]=\frac{1}{10} \text {. }
$$

\section{3. "Adjacency"}

The frequency with which a second gene occurs on a heterologous chromosome adjacent to the site of the first, should be lower if the first gene is at either end of a subset, termed a discontinuity (D), than if it is on an intermediate chromosome (I). Now,

$$
\begin{aligned}
P[1 \text { st gene at } \mathrm{D}] & =\frac{4}{10} ; \\
P[2 \mathrm{~A} / 1 \text { st gene at } \mathrm{D}] & =\frac{1}{10} ;
\end{aligned}
$$

and

$$
\begin{aligned}
P[1 \text { st gene at } \mathrm{I}] & =\frac{6}{16} ; \\
P[2 \mathrm{~A} / 1 \text { st gene at } \mathrm{I}] & =\frac{2}{10} .
\end{aligned}
$$

Thus

leaving

$$
P[2 \mathrm{~A}]=\frac{4}{10} \cdot \frac{1}{10}+\frac{6}{10} \cdot \frac{2}{10}=\frac{16}{100}
$$

$$
P[0 \mathrm{~L}, 0 \mathrm{H}, 0 \mathrm{~A}]=\frac{64}{i \overline{0}(\overline{)}}
$$

\section{Distribution of 3 paralogous genes}

Here the derivation of probabilities is based on the path coefficients in Figure 1B.

\subsection{Linkage}

$$
\begin{aligned}
& P[3 \mathrm{~L}]=1_{1}=10 \cdot 10^{-3}=\frac{1}{100} ; \\
& P[2 \mathrm{~L}]=1_{2}=10\left(\begin{array}{l}
3 \\
2
\end{array}\right) 9 \cdot 10^{-3}=\frac{27}{100} ; \\
& P[0 \mathrm{~L}]=1_{3}=10.9 .8 \cdot 10^{-3}=\frac{72}{100}
\end{aligned}
$$

\section{2. "Homoeology"}

$$
\begin{aligned}
& h_{1}=\frac{1}{9} ; \quad h_{2}=\frac{8}{9} ; \\
& h_{3}=\left(\begin{array}{l}
3 \\
2
\end{array}\right) \cdot \frac{1}{9}=\frac{1}{3} ; \quad h_{4}=\frac{2}{3} .
\end{aligned}
$$

From Figure 1B,

$$
\begin{aligned}
& P[2 \mathrm{H}]=1_{2} h_{1}+1_{3} h_{3}=0 \cdot 27 \\
& P[0 \mathrm{H}]=1_{1}+1_{2} h_{2}+1_{3} h_{4}=0 \cdot 73 .
\end{aligned}
$$

\section{3. "Adjacency"}

$$
\begin{aligned}
a_{1} & =P[2 \mathrm{~A}, 2 \mathrm{~L} \text { at } \mathrm{D}]+P[2 \mathrm{~A}, 2 \mathrm{~L} \text { at I }] \\
& =\frac{1}{8} \cdot \frac{4}{10}+\frac{2}{8} \cdot \frac{6}{10}=\frac{1}{5} ; a_{2}=1-a_{1}=\frac{4}{5} .
\end{aligned}
$$

Similarly

$$
a_{3}=\frac{2}{8} \cdot \frac{4}{10}+\frac{1}{2} \cdot \frac{6}{10}=\frac{2}{5} ; \quad a_{4}=\frac{3}{5} .
$$

In the absence of linkage and "homoeology" of the first two genes,

$P$ [all 3 genes in same subset of five heterologues $]=$

and so

$$
\frac{4}{8} \cdot \frac{3}{6}=\frac{1}{4}
$$

$$
P[2 \text { in one subset and } 1 \text { in the other }]=\frac{3}{4} \text {. }
$$

As there are three ways in which 3 of 5 sequentially arranged chromosomes can be adjacent,

$$
a_{5}=\frac{3}{\left(\begin{array}{l}
5 \\
3
\end{array}\right)} \cdot \frac{1}{4}=\frac{3}{40} \text {, }
$$

$$
\begin{aligned}
a_{6}= & P[2 \mathrm{~A}, 3 \text { in subset }] \\
& +\mathrm{P}[2 \mathrm{~A}, 2 \text { in subset }] \\
= & \frac{6}{\left(\begin{array}{l}
5 \\
3
\end{array}\right)} \cdot \frac{1}{4}+\frac{4}{\left(\begin{array}{l}
5 \\
2
\end{array}\right)} \cdot \frac{3}{4}=\frac{18}{40} . \\
a_{7}= & 1-a_{5}-a_{6}=\frac{19}{40} .
\end{aligned}
$$

From fig. $1 \mathrm{~B}$ we see that, $P[3 A]=1{ }_{3} h_{4} a_{5}=0.036$;

$P[2 \mathrm{~A}]=1_{2} \mathrm{~h}_{2} \mathrm{a}_{1}+1_{3} \mathrm{~h}_{3} \mathrm{a}_{3}+1_{3} \mathrm{~h}_{4} \mathrm{a}_{6}=0 \cdot 360$

and

$$
P[0 \mathrm{~A}]=1_{1}+1_{2} \mathrm{~h}_{1}+1_{2} \mathrm{~h}_{2} \mathrm{a}_{3}+1_{3} \mathrm{~h}_{3} \mathrm{a}_{4}+1_{3} \mathrm{~h}_{4} \mathrm{a}_{7}=0 \cdot 604 \text {. }
$$

Figure 1B Path coefficients $(l, h, a)$ determining the random distribution of 3 paralogous genes amongst the gametic complement of Zea mays arranged according to Bennett (1983)

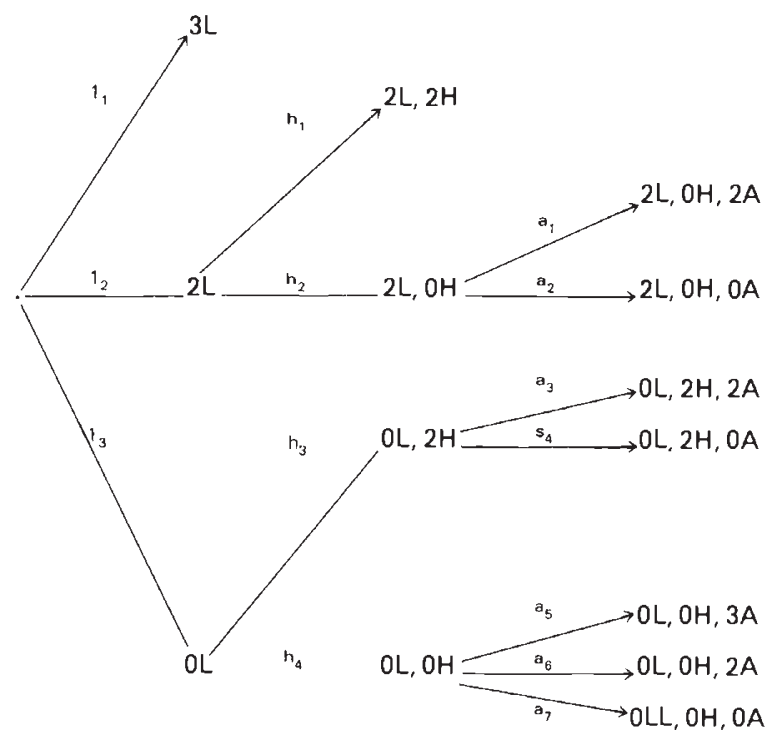

